\title{
Flatness-Based Adaptive Fuzzy Control of Autonomous Submarines
}

\author{
G. Rigatos ${ }^{1} \cdot$ P. Siano ${ }^{2}$
}

Received: 17 March 2015 / Revised: 19 August 2015 / Accepted: 28 August 2015 / Published online: 11 September 2015

(C) Springer Science+Business Media Singapore 2015

\begin{abstract}
The paper proposes adaptive fuzzy control based on differential flatness theory for autonomous submarines. It is proven that the dynamic model of the submarine, having as state variables the vessel's depth and its pitch angle, is a differentially flat one. This means that all its state variables and its control inputs can be written as differential functions of the flat output and its derivatives. By exploiting differential flatness properties the system's dynamic model is written in the multivariable linear canonical (Brunovsky) form, for which the design of a state feedback controller becomes possible. After this transformation, the new control inputs of the system contain unknown nonlinear parts, which are identified with the use of neurofuzzy approximators. The learning procedure for these estimators is determined by the requirement the first derivative of the closed-loop's Lyapunov function to be a negative one. Moreover, the Lyapunov stability analysis shows that H-infinity tracking performance is succeeded for the feedback control loop and this assures improved robustness to the aforementioned model uncertainty as well as to external perturbations. The efficiency of the proposed adaptive fuzzy control scheme is confirmed through simulation experiments.
\end{abstract}

Electronic supplementary material The online version of this article (doi:10.1007/s40903-015-0025-6) contains supplementary material, which is available to authorized users.

\section{G. Rigatos}

grigat@ieee.org

P. Siano

psiano@unisa.it

1 Unit of Industrial Automation, Industrial Systems Institute, 26504 Rion Patras, Greece

2 Department of Industrial Engineering, University of Salerno, 84084 Fisciano, Italy
Keywords Autonomous underwater vessels $\cdot$ Nonlinear control - Multivariable control - Nonlinear estimation . Adaptive fuzzy control · Differential flatness theory

\section{Introduction}

The design of control systems for autonomous underwater vessels (AUVs) and submarines is a non-trivial problem because such systems exhibit a highly nonlinear multivariable dynamics with strong couplings between their inputs and outputs [1-3]. Besides, such systems function under variable conditions and thus their dynamic model is subjected to parametric changes. Moreover, submersible autonomous robots and submarines are exposed to strong perturbations due to variable sea conditions and sea currents. Therefore, it is important to develop feedback control schemes for AUVs and submarines that will be little dependent on prior and exact knowledge of the associated dynamic model and will exhibit sufficient robustness to perturbation inputs [4-11]. To this end, in the recent years several research results have been presented, in particular on robust control [12-15] and on adaptive control of AUVs [16-19].

Adaptive fuzzy control methods can provide a solution to the problem of trajectory tracking and stabilization for autonomous submarines. Adaptive fuzzy control schemes have been developed for unknown single-input single-output (SISO) and unknown multi-input multi-output (MIMO) dynamical systems. The capability of neurofuzzy controllers to compensate for model parametric uncertainties, external disturbances, as well as for incomplete measurement of the systems state vector has been analyzed in several studies [2024]. Adaptive fuzzy control methods usually try to invert the system's dynamics, and thus to succeed convergence of its output to the desirable setpoints, starting from a description of 
the system in the canonical form. On the other hand, differential flatness theory enables to transform the system's generic description $x=f(x, u)$ into the canonical form and from that point on to develop adaptive control schemes. Consequently, differential flatness theory extends the class of nonlinear control systems to which adaptive neural / fuzzy control can be applied and this is a significant benefit for adaptive control theory [25-28].

In this paper, an adaptive control scheme is developed for autonomous submarines based on differential flatness theory and on real-time identification of the unknown dynamics of the system with the use of neurofuzzy approximators $[29,30]$. First, it is proven that the dynamic model of the submersible vessel, comprising as state variables the submarine's depth and its pitch angle, is a differentially flat one. This means that all its state variables and its control inputs can be expressed as differential functions of a specific algebraic variable which is the so-called flat output. By exploiting the differential flatness properties of the submarine's model its transformation into the linear canonical (Brunovsky) form is succeeded. For the latter description of the system's dynamics the design of a MIMO state feedback controller becomes possible [31-38]. In the transformed state-space model, the new control inputs of the submarine contain unknown nonlinear terms which are identified in real-time with the use of neurofuzzy approximators. The learning procedure for these estimators is determined by the requirement the first derivative of the closed-loop's Lyapunov function to be a negative one. Moreover, through Lyapunov stability analysis it is proven that the control system satisfies the H-infinity tracking conditions. This assures the control loop's robustness against model uncertainties and external perturbations. Finally, the efficiency of the submarine's control scheme is confirmed through simulation experiments.

The structure of the paper is as follows: in "The Dynamic Model of the Autonomous Submarine" section the nonlinear and multivariable dynamical model of the submarine is analyzed. In "Estimation of the Submarine's Unknown Dynamics" section the differential flatness properties of the submarine's model are proven and it is explained how one can perform estimation of the unknown dynamics of the submarine using neurofuzzy approximators. In "Flatness-based Adaptive Fuzzy Control of the Submarine Dynamics" section a flatness-based adaptive fuzzy controller is designed for the MIMO model of the autonomous submarine. In "Lyapunov Stability Analysis" section Lyapunov stability analysis is performed for the proposed submarine's control scheme and it is proven that the control loop satisfies the H-infinity tracking performance criterion. In "Simulation Tests" section simulation tests confirm the stability and robustness features of the submarine's control method. Finally, in "Conclusions" section concluding remarks are stated.
The Dynamic Model of the Autonomous Submarine

The dive-plane nonlinear time-varying dynamic model of the submarine is considered (see. Fig. 1). The primary variables of this model are: (i) the diving speed along the vessel's $z$ axis (in a body-fixed frame), (ii) the pitch angle $\theta$ formed between the horizontal reference axis (in an inertial reference frame) and the $x$-axis of the vessel (in the body-fixed frame) [16].

The equations of motion of the vessel are:

$$
\begin{aligned}
\dot{w}(t)= & \left.\frac{Z_{w}^{\prime} U}{L m_{3}^{\prime}} w(t)+\frac{1}{m_{3}^{\prime}} \dot{Z}_{\dot{\theta}}^{\prime}+m^{\prime}\right) U \dot{\theta}(t)+\frac{Z_{\dot{Q}}^{\prime} L}{m_{s}^{\prime}} \dot{Q}(t) \\
& +\frac{Z_{\delta B}^{\prime} U^{2}}{m_{3}^{\prime} L} \delta B(t)+\frac{Z_{\delta S}^{\prime} U^{2}}{m_{3}^{\prime} L} \delta S(t) \\
& +\frac{Z_{d}(t)}{0.5 \rho L^{3} m_{3}^{\prime}}+Z_{\eta}(w, q) \\
\dot{Q}(t)= & \frac{M_{\dot{w}}^{\prime}}{L I_{2}^{\prime}} \dot{w}(t)+\frac{M_{v}^{\prime} U}{L^{2} I_{2}^{\prime}} w(t)+\frac{M_{\dot{\theta}}^{\prime} U}{L I_{2}^{\prime}} \dot{\theta}(t) \\
& +\frac{M_{\delta B}^{\prime} U^{2}}{L^{2} I_{2}^{\prime}} \delta B(t)+\frac{M_{\delta S}^{\prime} U^{2}}{L^{2} I_{2}^{\prime}} \delta S(t) \\
& +\frac{2 m g\left(z_{G}-z_{B}\right)}{\rho L^{5} I_{2}^{\prime}} \theta(t)+\frac{M_{d}(t)}{0.5 \rho L^{5} I_{2}^{\prime}}+M_{\eta}(w, q)
\end{aligned}
$$

In the above dynamic model of the submarine $w$ is the velocity along the $z$-axis, $h$ is the depth of the vessel measured in the inertial coordinates system, $\theta$ is the pitch angle, $Q=\dot{\theta}$ is the rate of change of the pitch angle, $\delta B$ is the hydroplane deflection in the bow plane, $\delta S$ is the hydroplane deflection in the stern plane, and $Z_{d}, M_{d}$ are bounded disturbance inputs due to sea currents. Moreover, $Z_{\eta}(w, q), M_{\eta}(w, q)$ are disturbance inputs representing the vessel's cross-flow drag (the

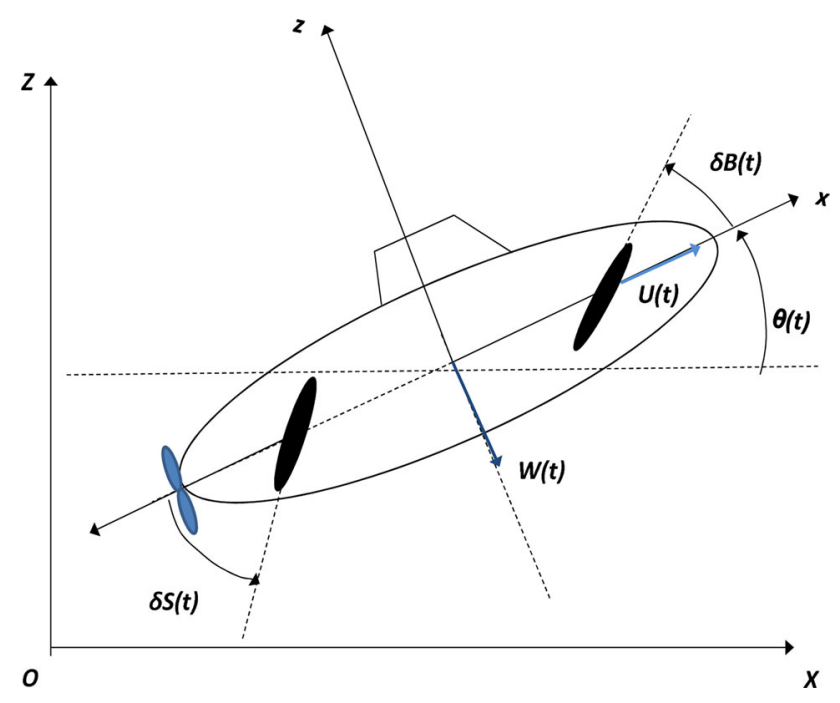

Fig. 1 Reference frames for the autonomous submarine 
Table 1 Parameters of the Submarine's dynamic model

\begin{tabular}{lll}
\hline Parameter value & Parameter value & Parameter value \\
\hline$Z_{w}^{\prime}=-0.0110$ & $Z_{\dot{w}}^{\prime}=-0.0075$ & $Z_{\theta}^{\prime}=-0.0045$ \\
$Z_{\theta}^{\prime}=-0.0002$ & $Z_{\delta B}^{\prime}=-0.0025$ & $Z_{\delta S}^{\prime}=-0.0050$ \\
$M_{w}^{\prime}=0.0030$ & $M_{\dot{w}}^{\prime}=-0.0002$ & $M_{\theta}^{\prime}=-0.0025$ \\
$M_{\dot{\theta}}^{\prime}=-0.0004$ & $M_{\delta B}^{\prime}=0.0005$ & $M_{\delta S}^{\prime}=-0.0025$ \\
$I_{y}^{\prime}=5.6867 \cdot 10 e^{-4}$ & $L=286 \mathrm{ft}$ & $m=1.52 \cdot 10^{5} \mathrm{slug}$ \\
$Z_{g}-Z_{B}=-1.5 \mathrm{ft}$ & $U=8.43 \mathrm{ft} / \mathrm{s}$ & $\rho=2.0 \mathrm{slug} / \mathrm{ft}^{3}$ \\
$I_{2}^{\prime}=I_{y}^{\prime}-M_{B}^{\prime}$ & $\left.m=2 m /\left(\rho L^{3}\right)\right)$ & $m_{3}^{\prime}=m^{\prime}-Z_{w}^{\prime}$ \\
\hline
\end{tabular}

latter is a function that contains the terms $w|w|$ and $Q|Q|$, as well as higher-order terms of $w$ and Q).

Actually, for the computation of the mathematical model of the vessel, the precise knowledge of the terms $Z_{\eta}(w, Q)$ and $M_{\eta}(w, Q)$ is not necessary since they can be treated by the adaptive control scheme as disturbances. The term $U=U_{0}$ denotes the $x$-axis (forward) velocity of the vessel.

The dynamic model of the submarine is completed by the following coefficients, given in Table 1 [16].

The control input of the submarine's model is described by the vector

$u=[\delta B(t) \delta S(t)]^{T}$

that is the control input consists of the hydroplane deflections in the bow and stern planes. A first description of the vessel's dynamics in matrix from is given by

$$
\left(\begin{array}{c}
\dot{w} \\
\dot{Q}
\end{array}\right)=\left(\begin{array}{c}
f_{W}(w, \theta, Q, t) \\
f_{\theta}(w, \theta, Q, t)
\end{array}\right)+B_{o} u
$$

$\dot{h}=w \cos (\theta)-U_{o} \sin (\theta) \Rightarrow$

$\ddot{h}=\dot{w} \cos (\theta)-w \sin (\theta) \dot{\theta}-U_{o} \cos (\theta) \dot{\theta} \Rightarrow$

$\ddot{h}=\dot{w} \cos (\theta)-w Q \sin (\theta)-U_{o} Q \cos (\theta)$

Moreover, solving with respect to $w$. from the first row of Eq. (7) one obtains

$w=\left(\cos (\theta)^{-1}\right)\left(\dot{h}+U_{o} \sin (\theta)\right)$

Additionally, from Eq. (4) one gets

$$
\begin{gathered}
\dot{w}=f_{w}(w, \theta, Q, t)+B_{o_{11}} u_{1}+B_{o_{12}} u_{2} \\
\dot{Q}=f_{\theta}(w, \theta, Q, t)+B_{o_{21}} u_{1}+B_{o_{22}} u_{2}
\end{gathered}
$$

Substituting Eq. (8) and the first row of Eq. (9) into the third row of Eq. (7) gives

$$
\begin{aligned}
\ddot{h}= & {\left[f_{w}(w, \theta, Q, t)+B_{o_{11}} u_{1}+B_{o_{12}} u_{2}\right] \cos (\theta) } \\
& -\frac{\left(\dot{h}+U_{0} \sin (\theta)\right)}{\cos (\theta)} Q \sin (\theta)-U_{0} Q \cos (\theta)
\end{aligned}
$$

Next by denoting

$$
\begin{aligned}
f_{w}(w, \theta, Q, t) & =g_{h}(h, \dot{h}, \theta, \dot{\theta}, t) \\
f_{\theta}(w, \theta, Q, t) & =g_{\theta}(h, \dot{h}, \theta, \dot{\theta}, t)
\end{aligned}
$$

from Eq. (10) and the second row of Eq. (9) one obtains

$$
\begin{aligned}
\ddot{h}= & g_{h}(h, \dot{h}, \theta, \dot{\theta}, t) \cos (\theta) \\
& -\frac{\left(\dot{h}+U_{0} \sin (\theta)\right)}{\cos (\theta)} \dot{\theta} \sin (\theta)-U_{0} \dot{\theta} \cos (\theta) \\
& +B_{0_{11} \cos (\theta) u_{1}+B_{0_{12}} \cos (\theta) u_{2}} \\
\ddot{\theta}= & g_{\theta}(h, \dot{h}, \theta, \dot{\theta}, t)+B_{0_{21}} u_{1}+B_{0_{22}} u_{2}
\end{aligned}
$$

where

$$
\left(\begin{array}{c}
f_{w}(w, \theta, Q, t) \\
f_{\theta}\left(f_{W}(w, \theta, Q, t)\right.
\end{array}\right)=M^{-1}\left(\begin{array}{c}
\left.\frac{Z_{w}^{\prime} U}{L m_{3}^{\prime}} w(t)+\frac{1}{m_{3}^{\prime}} \dot{Z}_{\dot{\theta}}^{\prime}+m^{\prime}\right) U \dot{\theta}(t)+\frac{Z_{\dot{Q}}^{\prime} L}{m_{s}^{\prime}} \dot{Q}(t)+\frac{Z_{d}(t)}{0.5 \rho L^{3} m_{3}^{\prime}}+Z_{\eta}(w, q) \\
\frac{M_{\dot{w}}^{\prime}}{L I_{2}^{\prime}} \dot{w}(t)+\frac{M_{v}^{\prime} U}{L^{2} I_{2}^{\prime}} w(t)+\frac{M_{\dot{\theta}}^{\prime} U}{L I_{2}^{\prime}} \dot{\theta}(t)+\frac{2 m g\left(z_{G}-z_{B}\right)}{\rho L^{5} I_{2}^{\prime}} \theta(t)+\frac{M_{d}(t)}{0.5 \rho L^{5} I_{2}^{\prime}}+M_{\eta}(w, q)
\end{array}\right)
$$

while for matrices $M$ and $B_{o}$ it holds

$$
M=\left(\begin{array}{cc}
1 & -Z_{\dot{Q}}^{L} / m_{3}^{\prime} \\
-M_{\ddot{w}}\left(L I_{2}^{\prime-1}\right) & 1
\end{array}\right) B_{O}=\left(\begin{array}{cc}
\frac{Z_{\delta B}^{\prime} U^{2}}{m_{3}^{\prime} L} & \frac{Z_{\delta S}^{\prime} U^{2}}{m_{3}^{\prime} L} \\
\frac{M^{\prime} \delta B U^{2}}{L^{2} I_{2}^{\prime}} & \frac{M^{\prime} \delta S U^{2}}{L^{2} I_{2}^{\prime}}
\end{array}\right)
$$

It holds that the depth of the vessel measured in the inertial reference frame and the velocity of the submarine along the $z$-axis of the body-fixed frame are related as follows:
Thus, from Eqs. (12) and (13) and by defining the state vector

$x=[h, \dot{h}, \theta, \dot{\theta}]^{T}$

the dynamic model of the submarine is written as

$$
\begin{aligned}
\left(\begin{array}{l}
\ddot{x}_{1} \\
\ddot{x}_{3}
\end{array}\right)= & \left(\begin{array}{l}
g_{b}(x, t) \cos \left(x_{3}\right)-\frac{x_{4}+U_{0} \sin \left(x_{3}\right)}{\cos \left(x_{3}\right)} x_{4} \sin \left(x_{3}\right)-U_{0} x_{4} \cos \left(x_{3}\right) \\
g_{\theta}(x, t)
\end{array}\right) \\
& +\left(\begin{array}{ll}
B_{0_{11}} & B_{0_{12}} \\
B_{0_{21}} & B_{0_{22}}
\end{array}\right)\left(\begin{array}{l}
u_{1} \\
u_{2}
\end{array}\right)
\end{aligned}
$$


or equivalently in the MIMO form

$\left(\begin{array}{l}\ddot{x}_{1} \\ \ddot{x}_{3}\end{array}\right)=\left(\begin{array}{l}f_{1}(x, t) \\ f_{2}(x, t)\end{array}\right)+\left(\begin{array}{ll}g_{11}(x, t) & g_{12}(x, t) \\ g_{21}(x, t) & g_{22}(x, t)\end{array}\right)\left(\begin{array}{l}u_{1} \\ u_{2}\end{array}\right)$

\section{Estimation of the Submarine's Unknown Dynamics}

\section{Differential Flatness of the Submarine's Model}

It can be proven that the submarine's MIMO nonlinear model given in Eq. (16) is a differentially flat one, having as flat output the vector

$y=\left[x_{1}, x_{3}\right]^{T}=[h, \theta]^{T}$

As explained above it holds that $x_{2}=\dot{x}_{1}$ and $x_{4}=\dot{x}_{3}$, which also means

$x_{2}=\left[\begin{array}{lll}1 & 0\end{array}\right] \dot{y}$

$x_{4}=\left[\begin{array}{ll}0 & 1\end{array}\right] \dot{y}$

Moreover, by solving Eq. (16) with respect to the control input $u$ one obtains

$$
\left(\begin{array}{l}
u_{1} \\
u_{2}
\end{array}\right)=\left(\begin{array}{ll}
g_{11}(x) & g_{12}(x) \\
g_{21}(x) & g_{22}(x)
\end{array}\right)^{-1}\left(\left(\begin{array}{l}
\ddot{x}_{1} \\
\ddot{x}_{2}
\end{array}\right)-\left(\begin{array}{l}
f_{1}(x) \\
f_{2}(x)
\end{array}\right)\right)
$$

and since the elements of the state vector $x$ are functions of the flat output, one has $u_{1}=f_{a}(y, \dot{y}, \ddot{y})$ and $u_{2}=f_{b}(y, \dot{y}, \ddot{y})$. Therefore, one finally has that all elements of the submarine's state vector and the control inputs can be written as functions of the flat output and its derivatives [31-38]. Consequently, the system is a differentially flat one.

By exploiting the differentially flat description of the system. the submarine's model can be written in the linear canonical (Brunovsky) form. To this end the following transformed control inputs are defined

$$
\begin{aligned}
& v_{1}=f_{1}(x, t)+g_{11} u_{1}+g_{12} u_{2} \\
& v_{2}=f_{2}(x, t)+g_{21} u_{1}+g_{22} u_{2}
\end{aligned}
$$

Therefore, one gets

$$
\left(\begin{array}{l}
\dot{y}_{1} \\
\dot{y}_{2} \\
\dot{y}_{3} \\
\dot{y}_{4}
\end{array}\right)=\left(\begin{array}{llll}
0 & 1 & 0 & 0 \\
0 & 0 & 0 & 0 \\
0 & 0 & 0 & 1 \\
0 & 0 & 0 & 0
\end{array}\right)\left(\begin{array}{l}
y_{1} \\
y_{2} \\
y_{3} \\
y_{4}
\end{array}\right)+\left(\begin{array}{ll}
0 & 0 \\
1 & 0 \\
0 & 0 \\
0 & 1
\end{array}\right)\left(\begin{array}{l}
v_{1} \\
v_{2}
\end{array}\right)
$$

while it is considered that the complete state vector of the submarine $y=[h, \dot{h}, \theta, \dot{\theta}]$ is available through measurements.

\section{Approximation of the Submarine's Unknown Dynamics}

The control signal of the MIMO nonlinear system which has been transformed into the Brunovsky form as described by Eq. (21) contains the unknown nonlinear functions $f(x)$ and $g(x)$ which can be approximated by

$\hat{f}\left(x \mid \theta_{f}\right)=\Phi_{f}(x) \theta_{f}$

where

$\Phi_{f}(x)=\left(\xi_{f}^{1}(x), \xi_{f}^{2}(x), \cdots \xi_{f}^{n}(x)\right)^{T}$

with $\xi_{f}^{i}(x), 1=1, \ldots, n$ being the vector of kernel functions (e.g. normalized fuzzy Gaussian membership functions), where

$\xi_{f}^{i}(x)=\left(\phi_{f}^{i, 1}(x), \phi_{f}^{i, 2}(x), \ldots, \phi_{f}^{i, N}(x)\right)$

thus giving

$\Phi_{f}(x)=\left(\begin{array}{cccc}\phi_{f}^{1,1}(x) & \phi_{f}^{1,2}(x) & \cdots & \phi_{f}^{1, N}(x) \\ \phi_{f}^{2,1}(x) & \phi_{f}^{2,2}(x) & \cdots & \phi_{f}^{2, N}(x) \\ \cdots & \cdots & \cdots & \cdots \\ \phi_{f}^{n, 1}(x) & \phi_{f}^{n, 2}(x) & \cdots & \phi_{f}^{n, N}(x)\end{array}\right)$

while the weights vector is defined as

$\theta_{f}^{T}=\left(\theta_{f}^{1}, \theta_{f}^{2}, \cdots \theta_{f}^{N}\right)$

$j=1, \ldots, N$ is the number of basis functions that is used to approximate the components of function $f$ which are denoted as $i=1, \ldots, n$. Thus, one obtains the relation of Eq. (22), i.e. $\hat{f}\left(x \mid \theta_{f}\right)=\Phi_{f}(x) \theta_{f}$.

In a similar manner, for the approximation of function $g$ one has

$\Phi_{g}(x)=\left(\xi_{g}^{1}(x), \xi_{g}^{2}(x), \cdots \xi_{g}^{N}(x)\right)^{T}$

with $\xi_{g}^{i}(x), 1=1, \ldots, N$ being the vector of kernel functions (e.g. normalized fuzzy Gaussian membership functions), where

$\xi_{g}^{i}(x)=\left(\phi_{g}^{i, 1}(x), \phi_{g}^{i, 2}(x), \cdots, \phi_{g}^{i, N}(x)\right)$

thus giving

$\Phi_{g}(x)=\left(\begin{array}{cccc}\phi_{g}^{1,1}(x) & \phi_{g}^{1,2}(x) & \cdots & \phi_{g}^{1, N}(x) \\ \phi_{g}^{2,1}(x) & \phi_{g}^{2,2}(x) & \cdots & \phi_{g}^{2, N}(x) \\ \cdots & \cdots & \cdots & \cdots \\ \phi_{g}^{n, 1}(x) & \phi_{g}^{n, 2}(x) & \cdots & \phi_{g}^{n, N}(x)\end{array}\right)$ 
while the weights vector is defined as

$\theta_{g}=\left(\theta_{g}^{1}, \theta_{g}^{2}, \cdots, \theta_{g}^{p}\right)^{T}$

where the components of matrix $\theta_{g}$ are defined as

$\theta_{g}^{j}=\left(\theta_{g_{1}}^{j}, \theta_{g_{2}}^{j}, \cdots \theta_{g_{N}}^{j}\right)$

$j=1, \ldots, N$ is the number of basis functions that is used to approximate the components of function $g$ which are denoted as $i=1, \ldots, n$. Thus one obtains about matrix $\theta_{g} \in R^{N \times p}$

$\theta_{g}=\left(\begin{array}{cccc}\theta_{g_{1}}^{1} & \theta_{g_{1}}^{2} & \cdots & \theta_{g_{1}}^{p} \\ \theta_{g_{2}}^{1} & \theta_{g_{2}}^{2} & \cdots & \theta_{g_{2}}^{p} \\ \cdots & \cdots & \cdots & \cdots \\ \theta_{g_{N}}^{1} & \theta_{g_{N}}^{2} & \cdots & \theta_{g_{N}}^{p}\end{array}\right)$

It holds that

$g=\left(\begin{array}{c}g_{1} \\ g_{2} \\ \cdots \\ g_{n}\end{array}\right)=\left(\begin{array}{cccc}g_{1}^{1} & g_{1}^{2} & \cdots & g_{1}^{p} \\ g_{2}^{1} & g_{2}^{2} & \cdots & g_{2}^{p} \\ \cdots & \cdots & \cdots & \ldots \\ g_{n}^{1} & g_{n}^{2} & \cdots & g_{n}^{p}\end{array}\right)$

Using the above, one finally has the relation of Eq. (22), i.e. $\hat{g}\left(x \mid \theta_{g}\right)=\Phi_{g}(x) \theta_{g}$. If the state variables of the system are available for measurement then a state-feedback control law can be formulated as

$u=\hat{g}^{-1}\left(x \mid \theta_{g}\right)\left[-\hat{f}\left(x \mid \theta_{f}\right)+y_{m}^{(r)}-K^{T} e+u_{c}\right]$

where $\hat{f}\left(x \mid \theta_{f}\right)$ and $\hat{g}\left(x \mid \theta_{g}\right)$ are fuzzy models to approximate $f(x)$ and $g(x)$, respectively. $u_{c}$ is a supervisory control term, e.g. $H_{\infty}$ control term that is used to compensate for the effects of modelling inaccuracies and external disturbances. Using the system's state-space description of Eq. (21) the control term $u_{c}$ is defined as

$u_{c}=-\frac{1}{r} B^{T} P e$

Moreover, $K^{T}$ is the feedback gain matrix that assures that the characteristic polynomial of the resulting closed-loop dynamics will be a Hurwitz one.

\section{Flatness-Based Adaptive Fuzzy Control of the Sub- marine Dynamics}

Next, taking into account also the effects of additive disturbances to the submarine the dynamic model of Eq. (16) becomes $\ddot{x}_{1}=f_{1}(x, t)+g_{1}(x, t) u+\tilde{d}_{1}$

$\ddot{x}_{3}=f_{2}(x, t)+g_{2}(x, t) u+\tilde{d}_{2}$

or, in matrix form

$\left(\begin{array}{l}\ddot{x}_{1} \\ \ddot{x}_{3}\end{array}\right)=\left(\begin{array}{l}f_{1}(x, t) \\ f_{2}(x, t)\end{array}\right)+\left(\begin{array}{l}g_{1}(x, t) \\ g_{2}(x, t)\end{array}\right) u+\left(\begin{array}{l}\tilde{d}_{1} \\ \tilde{d}_{2}\end{array}\right)$

The following control input is defined

$u=\left(\begin{array}{l}\hat{g}_{1}(x, t) \\ \hat{g}_{2}(x, t)\end{array}\right)^{-1} \cdot\left\{\left(\begin{array}{l}\ddot{x}_{1}^{d} \\ \ddot{x}_{3}^{d}\end{array}\right)-\left(\begin{array}{l}\hat{f}_{1}(x, t) \\ \hat{f}_{2}(x, t)\end{array}\right)-\left(\begin{array}{l}K_{1}^{T} \\ K_{2}^{T}\end{array}\right) e+\left(\begin{array}{l}u_{c_{1}} \\ u_{c_{2}}\end{array}\right)\right\}$

where $\left[u_{c_{1}} u_{c_{2}}\right]^{T}$ is a robust control term that is used for the compensation of the model's uncertainties as well as of the external disturbances and the vector of the feedback gain is $K_{i}^{T}=\left[k_{1}^{i}, k_{2}^{i}, \cdots, k_{n-1}^{i}, k_{n}^{i}\right]$.

Substituting Eq. (38) into Eq. (37) the closed-loop tracking error dynamics is obtained

$$
\begin{aligned}
& \left(\begin{array}{l}
\ddot{x}_{1} \\
\ddot{x}_{3}
\end{array}\right)=\left(\begin{array}{l}
f_{1}(x, t) \\
f_{2}(x, t)
\end{array}\right)+\left(\begin{array}{l}
g_{1}(x, t) \\
g_{2}(x, t)
\end{array}\right)\left(\begin{array}{l}
\hat{g}_{1}(x, t) \\
\hat{g}_{2}(x, t)
\end{array}\right)^{-1} \\
& \cdot\left\{\left(\begin{array}{l}
\ddot{x}_{1}^{d} \\
\ddot{x}_{3}^{d}
\end{array}\right)-\left(\begin{array}{l}
\hat{f}_{1}(x, t) \\
\hat{f}_{2}(x, t)
\end{array}\right)-\left(\begin{array}{l}
K_{1}^{T} \\
K_{2}^{T}
\end{array}\right) e+\left(\begin{array}{l}
u_{c_{1}} \\
u_{c_{2}}
\end{array}\right)\right\}+\left(\begin{array}{l}
\tilde{d}_{1} \\
\tilde{d}_{2}
\end{array}\right)
\end{aligned}
$$

Equation (39) can now be written as

$$
\begin{aligned}
& \left(\begin{array}{l}
\ddot{x}_{1} \\
\ddot{x}_{3}
\end{array}\right)=\left(\begin{array}{l}
f_{1}(x, t) \\
f_{2}(x, t)
\end{array}\right) \\
& +\left\{\left(\begin{array}{l}
g_{1}(x, t)-\hat{g}_{1}(x, t) \\
g_{2}(x, t)-\hat{g}_{2}(x, t)
\end{array}\right)+\left(\begin{array}{l}
\hat{g}_{1}(x, t) \\
\hat{g}_{2}(x, t)
\end{array}\right)\right\}\left(\begin{array}{l}
\hat{g}_{1}(x, t) \\
\hat{g}_{2}(x, t)
\end{array}\right) \\
& +\left\{\left(\begin{array}{l}
\ddot{x}_{1}^{d} \\
\ddot{x}_{3}^{d}
\end{array}\right)-\left(\begin{array}{l}
\hat{f}_{1}(x, t) \\
\hat{f}_{2}(x, t)
\end{array}\right)-\left(\begin{array}{l}
K_{1}^{T} \\
K_{2}^{T}
\end{array}\right) e+\left(\begin{array}{l}
u_{c_{1}} \\
u_{c_{2}}
\end{array}\right)\right\}+\left(\begin{array}{l}
\tilde{d}_{1} \\
\tilde{d}_{2}
\end{array}\right)
\end{aligned}
$$

and using Eq. (38) this results into

$$
\begin{aligned}
\left(\begin{array}{l}
\ddot{e}_{1} \\
\ddot{e}_{3}
\end{array}\right)= & \left(\begin{array}{l}
f_{1}(x, t)-\hat{f}_{1}(x, t) \\
f_{2}(x, t)-\hat{f}_{2}(x, t)
\end{array}\right) \\
& +\left(\begin{array}{l}
g_{1}(x, t)-\hat{g}_{1}(x, t) \\
g_{2}(x, t)-\hat{g}_{2}(x, t)
\end{array}\right) u \\
& -\left(\begin{array}{l}
K_{1}^{T} \\
K_{2}^{T}
\end{array}\right) e+\left(\begin{array}{l}
u_{c_{1}} \\
u_{c_{2}}
\end{array}\right)+\left(\begin{array}{l}
\tilde{d}_{1} \\
\tilde{d}_{2}
\end{array}\right)
\end{aligned}
$$

The following description for the approximation error is defined 


$$
\begin{aligned}
w= & \left(\begin{array}{l}
f_{1}(x, t)-\hat{f}_{1}(x, t) \\
f_{2}(x, t)-\hat{f}_{2}(x, t)
\end{array}\right) \\
& +\left(\begin{array}{l}
g_{1}(x, t)-\hat{g}_{1}(x, t) \\
g_{2}(x, t)-\hat{g}_{2}(x, t)
\end{array}\right) u
\end{aligned}
$$

Moreover, the following matrices are defined

$$
\begin{aligned}
A & =\left(\begin{array}{llll}
0 & 1 & 0 & 0 \\
0 & 0 & 0 & 0 \\
0 & 0 & 0 & 1 \\
0 & 0 & 0 & 0
\end{array}\right), \quad B=\left(\begin{array}{lll}
0 & 0 \\
1 & 0 \\
0 & 0 \\
0 & 1
\end{array}\right) \\
K^{T} & =\left(\begin{array}{llll}
K_{1}^{1} & K_{2}^{1} & K_{3}^{1} & K_{4}^{1} \\
K_{1}^{2} & K_{2}^{2} & K_{3}^{2} & K_{4}^{2}
\end{array}\right)
\end{aligned}
$$

Using matrices $A, B, K^{T}$, Eq. (41) is written in the following form

$$
\begin{aligned}
\dot{e}= & \left(A-B K^{T}\right) e+B u_{c}+B\left\{\left(\begin{array}{l}
f_{1}(x, t)-\hat{f}_{1}(x, t) \\
f_{2}(x, t)-\hat{f}_{2}(x, t)
\end{array}\right)\right. \\
& \left.+\left(\begin{array}{l}
g_{1}(x, t)-\hat{g}_{1}(x, t) \\
g_{2}(x, t)-\hat{g}_{2}(x, t)
\end{array}\right) u+\tilde{d}\right\}
\end{aligned}
$$

Next, the following approximators of the unknown system dynamics are defined

$\hat{f}(x)=\left(\begin{array}{llll}\hat{f}_{1}\left(x \mid \theta_{f}\right) & x \in R^{4 \times 1} & \hat{f}_{1}\left(x \mid \theta_{f}\right) & \in R^{1 \times 1} \\ \hat{f}_{2}\left(x \mid \theta_{f}\right) & x \in R^{4 \times 1} & \hat{f}_{2}\left(x \mid \theta_{f}\right) & \in R^{1 \times 1}\end{array}\right)$

with kernel functions

$\phi_{f}^{i, j}(x)=\frac{\prod_{j=1}^{n} \mu_{A_{j}}^{i}\left(x_{j}\right)}{\sum_{i=1}^{N} \prod_{j=1}^{n} \mu_{A_{j}}^{i}\left(x_{j}\right)}$

where $l=1,2$ and $\mu_{A_{j}^{i}}(x)$ is the $i$-th membership function of the antecedent (IF) part of the $l$-th fuzzy rule. Similarly, the following approximators of the unknown system dynamics are defined

$\hat{g}(x)=\left(\begin{array}{llll}\hat{g}_{1}\left(x \mid \theta_{g}\right) & x \in R^{4 \times 1} & \hat{g}_{1}\left(x \mid \theta_{g}\right) & \in R^{1 \times 2} \\ \hat{g}_{2}\left(x \mid \theta_{g}\right) & x \in R^{4 \times 1} & \hat{g}_{2}\left(x \mid \theta_{g}\right) & \in R^{1 \times 2}\end{array}\right)$

The values of the weights that result in optimal approximation are

$\theta_{f}^{*}=\arg \min _{\theta_{f} \in M_{\theta_{f}}}\left[\sup _{x \in U_{x}}\left(f(x)-\hat{f}\left(x \mid \theta_{f}\right)\right)\right]$

$\theta_{g}^{*}=\arg \min _{\theta_{g} \in M_{\theta g}}\left[\sup _{x \in U_{x}}\left(g(x)-\hat{g}\left(x \mid \theta_{g}\right)\right)\right]$

where the variation ranges for the weights are defined as

$M_{\theta_{f}}=\left\{\theta_{f} \in R^{h}:\left\|\theta_{f}\right\| \leq m_{\theta_{f}}\right\}$

$M_{\theta_{g}}=\left\{\theta_{g} \in R^{h}:\left\|\theta_{g}\right\| \leq m_{\theta_{g}}\right\}$
For the value of the approximation error defined in Eq. (42) that corresponds to the optimal values of the weights vectors $\theta_{f}^{*}$ and $\theta_{g}^{*}$ one has

$w=\left(f(x, t)-\hat{f}\left(x \mid \theta_{f}^{*}\right)\right)+\left(g(x, t)-\hat{g}\left(x \mid \theta_{g}^{*}\right)\right) u$

which is next written as

$$
\begin{aligned}
w= & \left(f(x, t)-\hat{f}\left(x \mid \theta_{f}\right)+\hat{f}\left(x \mid \theta_{f}\right)-\hat{f}\left(x \mid \theta_{f}^{*}\right)\right) \\
& +\left(g(x, t)-\hat{g}\left(x \mid \theta_{g}\right)+\hat{g}\left(x \mid \theta_{g}\right)-\hat{g}\left(x \mid \theta_{g}^{*}\right)\right) u
\end{aligned}
$$

which can be also written in the following form

$w=\left(w_{a}+w_{b}\right)$

where

$$
\begin{aligned}
& w_{a}=\left\{\left[\hat{f}\left(x, \theta_{f}\right)-\hat{f}\left(x \mid \theta_{f}^{*}\right)\right]+\left[\hat{g}\left(x, \theta_{g}\right)-\hat{g}\left(x \mid \theta_{g}^{*}\right)\right]\right\} \cdot u \\
& w_{b}=\left\{\left[f(x, t)-\hat{f}\left(x \mid \theta_{f}\right)\right]+\left[g(x, t)-\hat{g}\left(x \mid \theta_{g}\right)\right]\right\} u
\end{aligned}
$$

Moreover, the following weights error vectors are defined

$\tilde{\theta}_{f}=\theta_{f}-\theta_{f}^{*}$

$\tilde{\theta}_{g}=\theta_{g}-\theta_{g}^{*}$

Remark 1 The use of differential flatness theory enables to solve the problem of control of the nonlinear dynamics of the autonomous submarine in a conclusive manner: (i) by showing that a dynamical system is differentially flat it is possible to express its dynamics through specific primary variables which are the so-called flat outputs. All state variables of the system can be written as differential functions of the flat outputs, (ii) by showing that a dynamical system is differentially flat it can be assured that its transformation to the linear canonical (Brunovsky) form can be achieved, (iii) by expressing a differentially flat system into its equivalent linearized form the design of a state feedback controller for it can be completed in a few stages.

Remark 2 In case that the complete state vector of the submarine is not completely measurable one can implement an observer-based adaptive fuzzy control scheme based on differential flatness theory. The observer-based adaptive fuzzy control extends the class of systems to which indirect adaptive fuzzy control can be applied. This control method enables control of MIMO nonlinear systems without the need to measure all state vector elements [39]. The only assumption needed for the design of the observer-based controller and for succeeding $\mathrm{H}$-infinity tracking performance for the 
control loop is that there exists a solution for two Riccati equations associated with the linearized error dynamics of the differentially flat model. This assumption holds for several nonlinear systems, thus providing a systematic approach to the design of observer-based controllers.

\section{Lyapunov Stability Analysis}

\section{Stability Proof}

The following quadratic Lyapunov function is defined

$$
V=\frac{1}{2} e^{T} P e+\frac{1}{2 \gamma_{1}} \tilde{\theta}_{f}^{T} \tilde{\theta}_{f}+\frac{1}{2 \gamma_{2}} \operatorname{tr}\left[\tilde{\theta}_{g}^{T} \tilde{\theta}_{g}\right]
$$

Parameter $\gamma_{1}$ is the learning rate used in the adaptation of the weights of the neurofuzzy approximator for $f(x)$, while parameter $\gamma_{2}$ is the learning rate used in the adaptation of the weights of the neurofuzzy approximation for $g(x)$. It holds that

$$
\dot{V}=\frac{1}{2} \dot{e}^{T} P e+\frac{1}{2} e^{T} P \dot{e}+\frac{1}{\gamma_{1}} \dot{\tilde{\theta}}_{f}^{T} \tilde{\theta}_{f}+\frac{1}{\gamma_{2}} \operatorname{tr}\left[\dot{\tilde{\theta}}_{g}^{T} \tilde{\theta}_{g}\right]
$$

The tracking error dynamics is described by

$$
\begin{aligned}
\dot{e}= & \left(A-B K^{T}\right) e+B u_{c}+B\left\{\left(\begin{array}{l}
f_{1}(x, t)-\hat{f}_{1}(x, t) \\
f_{2}(x, t)-\hat{f}_{2}(x, t)
\end{array}\right)\right. \\
& \left.+\left(\begin{array}{l}
g_{1}(x, t)-\hat{g}_{1}(x, t) \\
g_{2}(x, t)-\hat{g}_{2}(x, t)
\end{array}\right) u+\tilde{d}\right\}
\end{aligned}
$$

and defining the approximation error

$$
\begin{aligned}
w= & \left(\begin{array}{l}
f_{1}(x, t)-\hat{f}_{1}(x, t) \\
f_{2}(x, t)-\hat{f}_{2}(x, t)
\end{array}\right) \\
& +\left(\begin{array}{l}
g_{1}(x, t)-\hat{g}_{1}(x, t) \\
g_{2}(x, t)-\hat{g}_{2}(x, t)
\end{array}\right) u
\end{aligned}
$$

the previous relation can be also written as

$$
\dot{e}=\left(A-B K^{T}\right) e+B u_{c}+B(w+\tilde{d})
$$

From Eq. (57) one obtains

$$
\begin{aligned}
\dot{V}= & \frac{1}{2}\left\{e^{T}\left(A-B K^{T}\right)^{T}+u_{c}^{T} B^{T}\right. \\
& \left.+(w+\tilde{d})^{T} B^{T}\right\} P e+\frac{1}{2} e^{T} P\left\{\left(A-B K^{T}\right) e\right. \\
& \left.+B u_{c}+B(w+\tilde{d})\right\}+\frac{1}{\gamma_{1}} \dot{\tilde{\theta}}_{f}^{T} \tilde{\theta}_{f}+\frac{1}{\gamma_{2}} \operatorname{tr}\left[\dot{\tilde{\theta}}_{g}^{T} \tilde{\theta}_{g}\right]
\end{aligned}
$$

which in turn gives

$$
\begin{aligned}
\dot{V}= & \frac{1}{2} e^{T}\left\{\left(A-B K^{T}\right)^{T} P+P\left(A-B K^{T}\right)\right\} e \\
& +\frac{1}{2} 2 e^{T} P B u_{c}+\frac{1}{2} 2 B^{T} P e(w+\tilde{d}) \\
& +\frac{1}{\gamma_{1}} \dot{\tilde{\theta}}_{f}^{T} \tilde{\theta}_{f}+\frac{1}{\gamma_{2}} \operatorname{tr}\left[\dot{\tilde{\theta}}_{g}^{T} \tilde{\theta}_{g}\right]
\end{aligned}
$$

Assumption 1 For given positive definite matrix $Q$ there exists a positive definite matrix $P$, which is the solution of the following matrix equation

$$
\begin{aligned}
& \left(A-B K^{T}\right)^{T} P+P\left(A-B K^{T}\right) \\
& -P B\left(\frac{2}{r}-\frac{1}{\rho^{2}}\right) B^{T} P+Q=0
\end{aligned}
$$

Substituting Eqs. (63) and (35) into $\dot{V}$ yields after some operations

$$
\begin{aligned}
\dot{V}= & \frac{1}{2} e^{T}\left\{-Q+P B\left(\frac{2}{r}-\frac{1}{\rho^{2}}\right) B^{T} P\right\} e \\
& +e^{T} P B\left\{-\frac{1}{r} B^{T} P e\right\}+B^{T} P(w+\tilde{d})+\frac{1}{\gamma_{1}} \dot{\tilde{\theta}}_{f}^{T} \tilde{\theta}_{f} \\
& +\frac{1}{\gamma_{2}} \operatorname{tr}\left[\dot{\tilde{\theta}}_{g}^{T} \tilde{\theta}_{g}\right]
\end{aligned}
$$

Therefore it holds

$$
\begin{aligned}
\dot{V}= & -\frac{1}{2} e^{T} Q e-\frac{1}{2 \rho^{2}} e^{T} P B B^{T} P e+e^{T} P B(w+\tilde{d}) \\
& +\frac{1}{\gamma_{1}} \dot{\tilde{\theta}}_{f}^{T} \tilde{\theta}_{f}+\frac{1}{\gamma_{2}} \operatorname{tr}\left[\dot{\tilde{\theta}}_{g}^{T} \tilde{\theta}_{g}\right]
\end{aligned}
$$

It also holds that

$$
\begin{gathered}
\dot{\tilde{\theta}}_{f}=\dot{\theta}_{f}-\dot{\theta}_{f}^{*}=\dot{\theta}_{f} \\
\dot{\tilde{\theta}}_{g}=\dot{\theta}_{g}-\dot{\theta}_{g}^{*}=\dot{\theta}_{g}
\end{gathered}
$$

The following weights adaptation law is used (Fig. 2)

$$
\begin{aligned}
\dot{\theta}_{f} & =-\gamma_{1} \Phi(x)^{T} B^{T} P e \\
\dot{\theta}_{g} & =-\gamma_{2} \Phi(x)^{T} B^{T} P e u^{T}
\end{aligned}
$$

This is a gradient-type learning method for the weights of the neurofuzzy approximators [40-42]. Assuming $N$ fuzzy rules and associated kernel functions the matrices dimensions are $\theta_{f} \in R^{N \times 1}, \theta_{g} \in R^{N \times 2}, \Phi(x) \in R^{2 \times N}, B \in R^{4 \times 2}, P \in R^{4 \times 4}$ and $e \in R^{4 \times 1}$. Therefore it holds

$$
\begin{aligned}
\dot{V}= & -\frac{1}{2} e^{T} Q e-\frac{1}{2 \rho^{2}} e^{T} P B B^{T} P e \\
& +e^{T} P B(w+\tilde{d})
\end{aligned}
$$




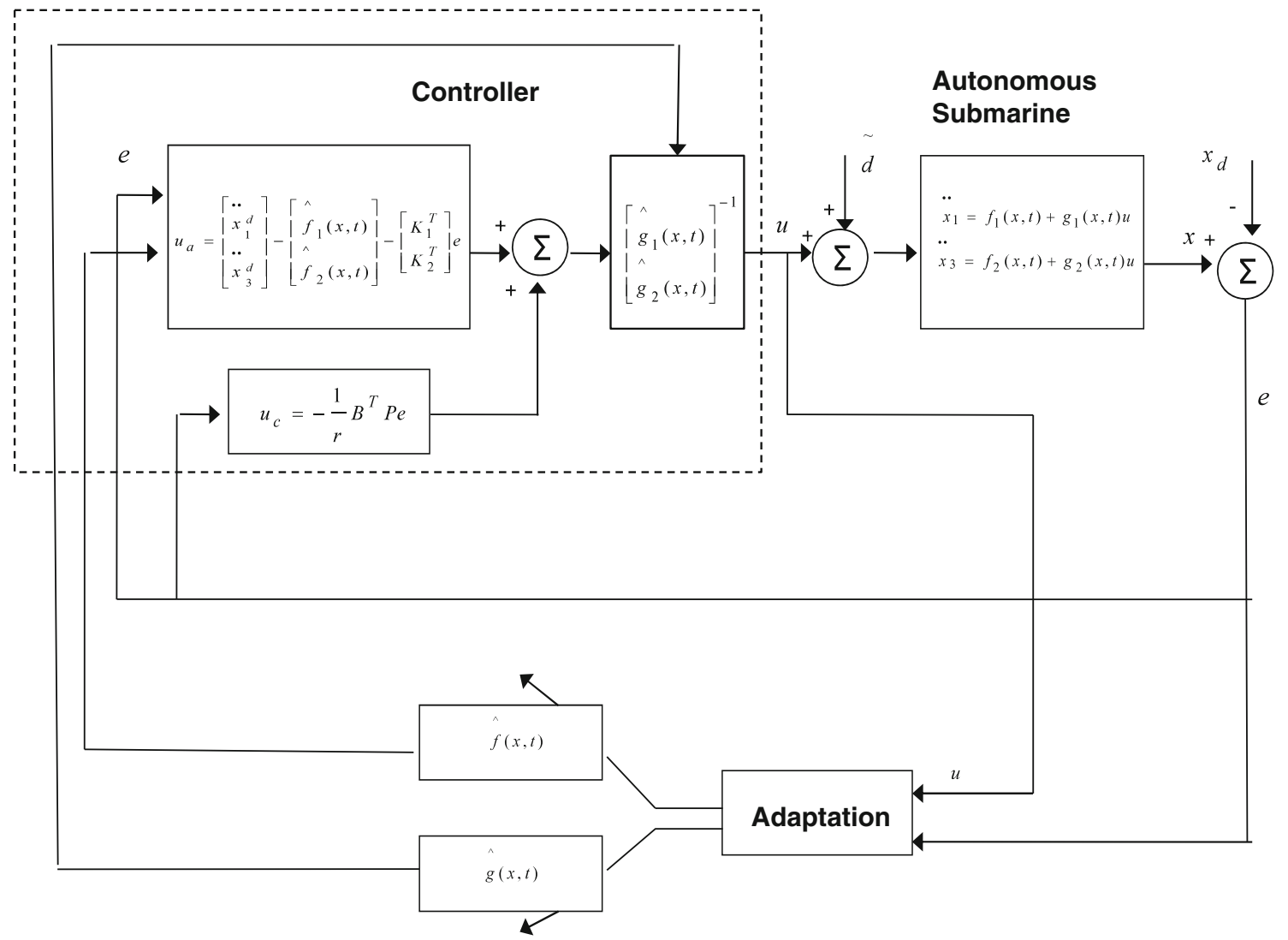

Fig. 2 Diagram of the flatness-based adaptive fuzzy controller for the autonomous submarine

$$
\begin{aligned}
& +\frac{1}{\gamma_{1}}\left(-\gamma_{1}\right) e^{T} P B \Phi(x)\left(\theta_{f}-\theta_{f}^{*}\right) \\
& +\frac{1}{\gamma_{2}}\left(-\gamma_{2}\right) \operatorname{tr}\left[u e^{T} P B \Phi(x)\left(\theta_{g}-\theta_{g}^{*}\right)\right]
\end{aligned}
$$

or

$$
\begin{aligned}
\dot{V}= & -\frac{1}{2} e^{T} Q e-\frac{1}{2 \rho^{2}} e^{T} P B B^{T} P e+e^{T} P B(w+\tilde{d}) \\
& +\frac{1}{\gamma_{1}}\left(-\gamma_{1}\right) e^{T} P B \Phi(x)\left(\theta_{f}-\theta_{f}^{*}\right) \\
& +\frac{1}{\gamma_{2}}\left(-\gamma_{2}\right) \operatorname{tr}\left[u e^{T} P B\left(\hat{g}\left(x \mid \theta_{g}\right)-\hat{g}\left(x \mid \theta_{g}^{*}\right)\right]\right.
\end{aligned}
$$

Taking into account that $u \in R^{2 \times 1}$ and $e^{T} P B\left(\hat{g}\left(x \mid \theta_{g}\right)-\right.$ $\left.\hat{g}\left(x \mid \theta_{g}^{*}\right)\right) \in R^{1 \times 2}$ it holds

$$
\begin{aligned}
\dot{V}= & -\frac{1}{2} e^{T} Q e-\frac{1}{2 \rho^{2}} e^{T} P B B^{T} P e+e^{T} P B(w+\tilde{d}) \\
& +\frac{1}{\gamma_{1}}\left(-\gamma_{1}\right) e^{T} P B \Phi(x)\left(\theta_{f}-\theta_{f}^{*}\right) \\
& +\frac{1}{\gamma_{2}}\left(-\gamma_{2}\right) \operatorname{tr}\left[e^{T} P B\left(\hat{g}\left(x \mid \theta_{g}\right)-\hat{g}\left(x \mid \theta_{g}^{*}\right)\right) u\right]
\end{aligned}
$$

Since $e^{T} P B\left(\hat{g}\left(x \mid \theta_{g}\right)-\hat{g}\left(x \mid \theta_{g}^{*}\right)\right) u \in R^{1 \times 1}$ it holds

$$
\begin{aligned}
& \operatorname{tr}\left(e^{T} P B\left(\hat{g}\left(x \mid \theta_{g}\right)-\hat{g}\left(x \mid \theta_{g}^{*}\right) u\right)\right. \\
& \quad=e^{T} P B\left(\hat{g}\left(x \mid \theta_{g}\right)-\hat{g}\left(x \mid \theta_{g}^{*}\right)\right) u
\end{aligned}
$$

Therefore, one finally obtains

$$
\begin{aligned}
\dot{V}= & -\frac{1}{2} e^{T} Q e-\frac{1}{2 \rho^{2}} e^{T} P B B^{T} P e+e^{T} P B(w+\tilde{d}) \\
& +\frac{1}{\gamma_{1}}\left(-\gamma_{1}\right) e^{T} P B \Phi(x)\left(\theta_{f}-\theta_{f}^{*}\right) \\
& +\frac{1}{\gamma_{2}}\left(-\gamma_{2}\right) e^{T} P B\left(\hat{g}\left(x \mid \theta_{g}\right)-\hat{g}\left(x \mid \theta_{g}^{*}\right)\right) u
\end{aligned}
$$

Next the following approximation error is defined

$$
w_{\alpha}=\left[\hat{f}\left(x \mid \theta_{f}\right)-\hat{f}\left(x \mid \theta_{f}^{*}\right)\right]+\left[\hat{g}\left(x \mid \theta_{g}\right)-\hat{g}\left(x \mid \theta_{g}^{*}\right)\right] u
$$

Thus, one obtains

$$
\begin{aligned}
\dot{V}= & -\frac{1}{2} e^{T} Q e-\frac{1}{2 \rho^{2}} e^{T} P B B^{T} P e \\
& +e^{T} P B(w+\tilde{d})+e^{T} P B w_{\alpha}
\end{aligned}
$$


Denoting the aggregate approximation error and disturbances vector as

$w_{1}=w+\tilde{d}+w_{\alpha}$

the derivative of the Lyapunov function becomes

$\dot{V}=-\frac{1}{2} e^{T} Q e-\frac{1}{2 \rho^{2}} e^{T} P B B^{T} P e+e^{T} P B w_{1}$

which in turn is written as

$$
\begin{aligned}
\dot{V}= & -\frac{1}{2} e^{T} Q e-\frac{1}{2 \rho^{2}} e^{T} P B B^{T} P e \\
& +\frac{1}{2} e^{T} P B w_{1}+\frac{1}{2} w_{1}^{T} B^{T} P e
\end{aligned}
$$

Next, a Lemma is introduced:

Lemma The following inequality holds:

$$
\frac{1}{2} e^{T} P B w_{1}+\frac{1}{2} w_{1}^{T} B^{T} P e-\frac{1}{2 \rho^{2}} e^{T} P B B^{T} P e \leq \frac{1}{2} \rho^{2} w_{1}^{T} w_{1}
$$

Proof The binomial $\left(\rho a-\frac{1}{\rho} b\right)^{2} \geq 0$ is considered. Expanding the left part of the above inequality one gets

$$
\begin{aligned}
\rho^{2} a^{2}+\frac{1}{\rho^{2}} b^{2}-2 a b \geq 0 & \Rightarrow \frac{1}{2} \rho^{2} a^{2}+\frac{1}{2 \rho^{2}} b^{2}-a b \geq 0 \Rightarrow \\
a b-\frac{1}{2 \rho^{2}} b^{2} \leq \frac{1}{2} \rho^{2} a^{2} & \Rightarrow \frac{1}{2} a b+\frac{1}{2} a b-\frac{1}{2 \rho^{2}} b^{2} \leq \frac{1}{2} \rho^{2} a^{2}
\end{aligned}
$$

The following substitutions are carried out: $a=w_{1}$ and $b=e^{T} P B$ and the previous relation becomes

$$
\frac{1}{2} w_{1}^{T} B^{T} P e+\frac{1}{2} e^{T} P B w_{1}-\frac{1}{2 \rho^{2}} e^{T} P B B^{T} P e \leq \frac{1}{2} \rho^{2} w_{1}^{T} w_{1}
$$

The previous inequality is used in $\dot{V}$, and the right part of the associated inequality is enforced

$\dot{V} \leq-\frac{1}{2} e^{T} Q e+\frac{1}{2} \rho^{2} w_{1}^{T} w_{1}$

As in the SISO systems case, the attenuation coefficient $\rho$ can be chosen such that the right part of Eq. (81) is always upper bounded by 0 . For instance, it suffices at every iteration of the control algorithm to have

$$
\begin{gathered}
-\frac{1}{2} e^{T} Q e+\frac{1}{2} \rho^{2}\left\|w_{1}\right\|^{2} \leq 0 \Rightarrow-\frac{1}{2}\|e\|_{Q}^{2}+\frac{1}{2} \rho^{2}\left\|w_{1}\right\|^{2} \leq 0 \\
\Rightarrow \frac{1}{2} \rho^{2}\left\|w_{1}\right\|^{2} \leq \frac{1}{2}\|e\|_{Q}^{2}+\Rightarrow \rho^{2} \leq \frac{\|e\|_{Q}^{2}}{\left\|w_{1}\right\|^{2}}
\end{gathered}
$$

Again without knowledge of the uncertainties and disturbance term $\left\|w_{1}\right\|$ a sufficiently small value of $\rho$ can assure that the above inequality holds and thus that the loop's stability is assured. Actually, $\rho$ should be given the least value which permits to obtain a solution of the Riccati equation (63).

Equation (81) can be used to show that the $H_{\infty}$ performance criterion is satisfied. The integration of $\dot{V}$ from 0 to $T$ gives

$$
\begin{aligned}
\int_{0}^{T} \dot{V}(t) d t \leq & -\frac{1}{2} \int_{0}^{T}\|e\|^{2} d t+\frac{1}{2} \rho^{2} \int_{0}^{T}\left\|w_{1}\right\|^{2} d t \\
\Rightarrow & 2 V(T)+\int_{0}^{T}\|e\|_{Q}^{2} d t \leq 2 V(0) \\
& +\rho^{2} \int_{0}^{T}\left\|w_{1}\right\|^{2} d t
\end{aligned}
$$

Moreover, if there exists a positive constant $M_{w}>0$ such that

$\int_{0}^{\infty}\left\|w_{1}\right\|^{2} d t \leq M_{w}$

then one gets

$\int_{0}^{\infty}\|e\|_{Q}^{2} d t \leq 2 V(0)+\rho^{2} M_{w}$

Thus, the integral $\int_{0}^{\infty}\|e\|_{Q}^{2} d t$ is bounded and according to Barbalat's Lemma one obtains $\lim _{t \rightarrow \infty} e(t)=0$.

\section{The Role of Riccati Equation Coefficients in $H_{\infty}$ Control Robustness}

The linearized tracking error dynamics of Eq. (60) is considered again, i.e.

$\dot{e}=\left(A-B K^{T}\right) e+B u_{c}+B(w+\tilde{d})$

The aim of $H_{\infty}$ control is to eliminate the impact of the modelling errors $w=[f(x, t)-\hat{f}(x, t)]+[g(x, t)-$ $\hat{g}(x, t)] u$ and the external disturbances $\tilde{d}$ which are not white noise signals. This implies the minimization of the quadratic cost function [43-45]:

$$
\begin{aligned}
J(t)= & \frac{1}{2} \int_{0}^{T} e^{T}(t) e(t)+r u_{c}^{T}(t) u_{c}(t) \\
& -\rho^{2}(w+\tilde{d})^{T}(w+\tilde{d}) d t, \quad r, \rho>0
\end{aligned}
$$

The weight $r$ determines how much the control signal should be penalized and the weight $\rho$ determines how much the disturbances influence should be rewarded in the sense of a mini-max differential game. The $H_{\infty}$ control law is 
$u(t)=-\frac{1}{r} B^{T} P e(t)$ where $P$ is the positive definite symmetric matrix derived from the algebraic Riccati equation Eq. (63).

The parameter $\rho$ in Eq. (86), is an indication of the closedloop system robustness. If the values of $\rho>0$ are excessively decreased with respect to $r$, then the solution of the Riccati equation is no longer a positive definite matrix. Consequently there is a lower bound $\rho_{\text {min }}$ of $\rho$ for which the $H_{\infty}$ control problem has a solution. The acceptable values of $\rho$ lie in the interval $\left[\rho_{\min }, \infty\right)$. If $\rho_{\text {min }}$ is found and used in the design of the $H_{\infty}$ controller, then the closed-loop system will have increased robustness. Unlike this, if a value $\rho>\rho_{\text {min }}$ is used, then an admissible stabilizing $H_{\infty}$ controller will be derived but it will be a suboptimal one. The Hamiltonian matrix

$H=\left(\begin{array}{cc}A & -\left(\frac{2}{r}-\frac{1}{\rho^{2}}\right) B B^{T} \\ -Q & -A^{T}\end{array}\right)$

provides a criterion for the existence of a solution of the Riccati equation Eq. (63). A necessary condition for the solution of the algebraic Riccati equation to be a positive semi-definite symmetric matrix is that $H$ has no imaginary eigenvalues [43].

Remark 3 Several methods on adaptive neural/fuzzy control of nonlinear dynamical systems assume that the system is already described in the canonical (Brunovsky form). However, for the majority of electromechanical systems this assumption does not hold. For example, robotic systems, UAVs (unmanned aerial vehicles), land vehicles and ship models, electric power generators of the PMSG and DFIG type, electric motors of the DC or induction type, power electronics models such as voltage converters are some examples of dynamical systems which are not inherently found in the canonical form [28,29]. The new method on differential flatness theory-based adaptive fuzzy control comes to address this issue. The proposed method, having as particular application example the motion control of the autonomous submarine, exhibits specific advantages comparing to other adaptive fuzzy and neural control methods. These have been explained in [28] and are summarized as follows: (i) The proposed control method achieves excellent accuracy in the tracking of the reference setpoints. This is a confirmation of the stability analysis that results in an $\mathrm{H}$-infinity tracking performance criterion and in an asymptotic stability condition. Moreover, it has been shown that the control input exhibits smooth variations. This is an advantage comparing, for instance, to adaptive fuzzy sliding-mode control since in the latter case there are abrupt control input variations (ii) The proposed Differential flatness theory approach to adaptive fuzzy control of MIMO nonlinear dynamical systems outperforms all other adaptive neural/fuzzy control methods because it can be applied to a wider class of nonlinear dynam- ical systems. The method can be applied to any type of MIMO differentially flat systems that admits a transformation to the canonical Brunovsky form and does not require the systems dynamic model to have a particular structure (e.g. affinein-the-input, triangular or other). Additional results about adaptive fuzzy controllers which are based on transformations into canonical forms can be found in [25-27].

Remark 4 In flatness-based control global linearization of the system's dynamics is performed by applying a change of coordinates (diffeomorphism) which is based on differential flatness theory. Actually, by expressing all state variables of the submarine's model as differential functions of primary variables which are called flat outputs one can arrive at an input-output linearized form of the submarines model for which the design of a state-feedback controller becomes possible. The following should be pointed out: (1) The proposed linearization method is valid in the entire state-space of the submarine's model, and this is why it is a global linearization method. On the other hand, in local linearization methods which are performed round an equilibrium and which require the computation of Jacobian matrices, the linearization remains valid only within a small radius round the equilibrium, (2) The proposed linearization method can be applied to all differentially flat systems. This is actually a very wide class of systems which comprises both models subjected to static feedback linearization and models that admit only dynamic feedback linearization. Once it is proven that an autonomous underwater vessel (AUV) satisfies differential flatness properties its transformation to the linear canonical form becomes possible and the design of a statespace controller is also feasible (3) Comparing to other global linearization approaches, such as the ones related to Lie algebra, linearization with the use of differential flatness theory is of wider scope $[46,47]$. In the case of Lie algebra, necessary and sufficient conditions for achieving linearization exist if a system is input-to-state linearizable. For systems which are input-output linearizable, the previous mentioned linearization conditions can be used only if the relative degree of the system is equal to the dimension of the system. Therefore, one cannot confirm all cases in which a system is input-output linearizable. On the contrary, by applying differential flatness theory and by proving that a system is differential flat one can also ascertain that the system is input-output linearizable, and can design a state feedback controller.

Remark 5 The contribution of adaptive fuzzy control based on differential flatness theory is that it is a completely modelfree control method. When designing the controller, there is no need for prior knowledge of the systems parameters and state-space equation. The only assumption made by the control method is that the systems dynamics is expressed in the generic form $\dot{x}=f(x, u)$ with $f$ being an unknown function. The control method is based on the online approximation 
of the unknown systems dynamics which is performed by neuro-fuzzy approximators. This information is used to compute the control input that is finally exerted on the system. Moreover, to compensate for modelling inaccuracies and external perturbations the control input is complemented by an H-infinity control term. Through Lyapunov stability analysis and from the requirement to a have always a negative sign for the first order derivative of the Lyapunov function, the learning rate of the neurofuzzy approximators is chosen. The stability analysis demonstrates, that despite having no prior knowledge of the systems dynamics, the adaptive fuzzy control scheme based on differential flatness theory assures the convergence of the state vector elements of the system to the desirable setpoints.

\section{Simulation Tests}

The results about the stability and robustness features of the submarine's control loop were also confirmed through simulation experiments. In the simulation tests, the dynamic model of the submarine was considered to be completely unknown and was identified in real-time by the previously analyzed neurofuzzy approximators. The estimated unknown dynamics of the system was used in the computation of the control inputs which were finally exerted on the submarine's model. The sampling period was set to $T_{s}=0.01 \mathrm{sec}$. Apart from modelling uncertainty it was considered that the submarine's model was also affected by external perturbations. The numerical values of the gains which have been used in the solution of the Riccati equation have been defined as $r=0.1$ and $\rho=1.0$.

The state feedback gain was $K \in R^{2 \times 4}$. The basis functions used in the estimation of $f_{i}(x, t), i=1,2$ and $g_{i j}(x, t), i=$ $1,2, j=1,2$ were $\mu_{A_{j}}(\hat{x})=e^{\left(\frac{\hat{x}-c j}{\sigma}\right)^{2}}, j=1, \ldots, 3$. Since there are four inputs $x_{1}, x_{2}$ and $x_{4}, x_{4}$ and the associated definition set (universe of discourse) consists of 3 fuzzy sets, for the approximation of functions $f_{i}(x, t) i=1,2$, there will be 81 fuzzy rules of the form:

$R^{l}: I F \quad x_{1}$ is $A_{1}^{l} A N D x_{2}$ is $A_{2}^{l}$

$A N D x_{3}$ is $A_{3}^{l} A N D x_{4}$ is $A_{4}^{l}$ THEN $\hat{f}_{i}^{l}$ is $b^{l}$

and $\hat{f}_{i}(x, t)=\frac{\sum_{l=1}^{81} \hat{f}_{i}^{l} \prod_{i=1}^{4} \mu_{A_{i}}^{l}\left(x_{i}\right)}{\sum_{l=1}^{81} \prod_{i=1}^{4} \mu_{A_{i}}^{l}\left(x_{i}\right)}$. Indicative (dimensionless) values for the placement on a spatial grid of the centers for the centers $c_{i}^{(l)}, i=1, \ldots, 4$ and the variances $v^{(l)}$ of each rule are as follows (Table 2).

As noted, in the considered fuzzy rule-base there are four input parameters in the antecedent parts of the fuzzy rules, i.e. $x_{1}=h, x_{2}=\dot{h}, x_{3}=\theta$ and $x_{4}=\dot{\theta}$. Each parameter is partitioned into three fuzzy sets. Therefore, by taking all possible
Table 2 Parameters of the fuzzy rule base

\begin{tabular}{llllll}
\hline Rule & $c_{1}^{(l)}$ & $c_{2}^{(l)}$ & $c_{3}^{(l)}$ & $c_{4}^{(l)}$ & $v^{(l)}$ \\
\hline$R^{(1)}$ & -1.0 & -1.0 & -1.0 & -1.0 & 3 \\
$R^{(2)}$ & -1.0 & -1.0 & -1.0 & 0.0 & 3 \\
$R^{(3)}$ & -1.0 & -1.0 & -1.0 & 1.0 & 3 \\
$R^{(4)}$ & -1.0 & -1.0 & 0.0 & -1.0 & 3 \\
$R^{(5)}$ & -1.0 & -1.0 & 0.0 & 0.0 & 3 \\
$R^{(6)}$ & -1.0 & -1.0 & 0.0 & 1.0 & 3 \\
$\ldots$ & $\ldots$ & $\ldots$ & $\ldots$ & $\ldots$ & $\ldots$ \\
$\ldots$ & $\ldots$ & $\ldots$ & $\ldots$ & $\ldots$ & $\ldots$ \\
$R^{(81)}$ & 1.0 & 1.0 & 1.0 & 0.5 & 3 \\
\hline
\end{tabular}

combinations between the fuzzy sets one has $3^{4}=81$ fuzzy rules. The finer the partition of the input variables into fuzzy sets is, the more accurate the approximation of the nonlinear system dynamics by the neuro-fuzzy model is expected to be (although some of the rules of the fuzzy rule base may not be sufficiently activated due to little coverage of the associated region of the patterns space by input data). However, considering a large number of fuzzy sets for each input variable induces the curse of dimensionality which means that there is an excessive and rather unnecessary increase in the number of the adaptable parameters that constitute the neuro-fuzzy model.

The associated results are presented in Figs. 3, 4 and 5. It can be observed that the adaptive fuzzy control scheme succeeded fast and accurate tracking of the reference setpoints. After finding the solution of the algebraic Riccati equation given in Eq. (63) the computation of an H-infinity feedback control term was possible and this provided the submarine's control loop with additional robustness. Taking into account that in real operating conditions the control of a submarine cannot be based on the assumption about a precise mathematical model and about complete knowledge of external perturbations, the significance of the proposed adaptive fuzzy control scheme becomes obvious.

Remark 6 There have been numerous examples of the use of model-based flatness-based control, given in [28,29]. If the model of the control system is a precise one flatness-based control is anticipated to have an excellent performance. The control problem becomes more complicated in the case of absence of a precise mathematical model for the controlled system. It is even more difficult when there is no prior knowledge about the systems dynamics that can be used in the design of the flatness-based controller. The solution to the latter control problem is obtained with the use of the proposed flatness-based adaptive fuzzy control method. Although the dynamic model of the system is completely unknown, it is assured through Lyapunov stability analysis that this uncer- 
Fig. 3 Setpoint 2: a convergence of the state variables $x_{i}, i=1, \ldots, 4$ of the submarine to the desirable setpoints, $\mathbf{b}$ variations of the control inputs (bow and stern hydroplane reflections)
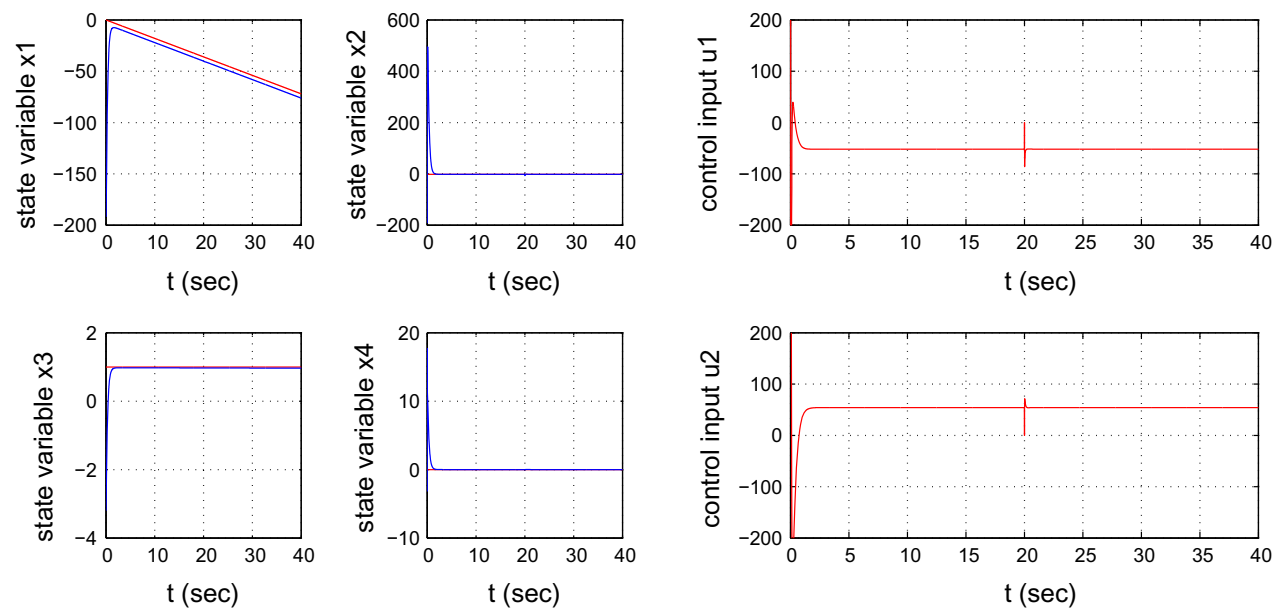

(a)

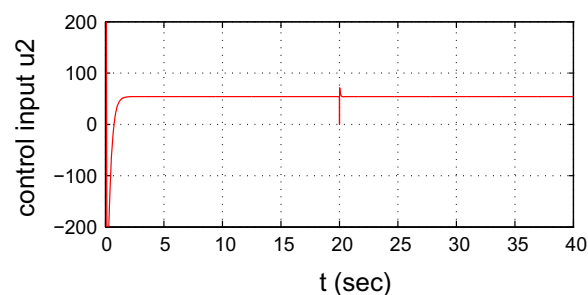

(b)
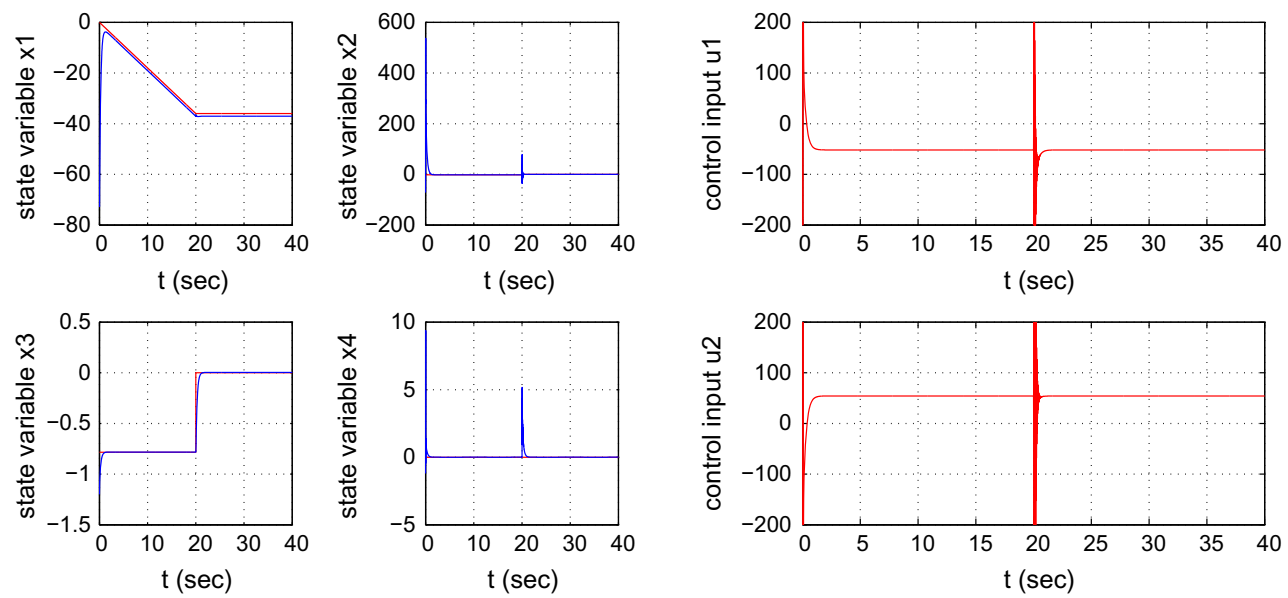

(a)

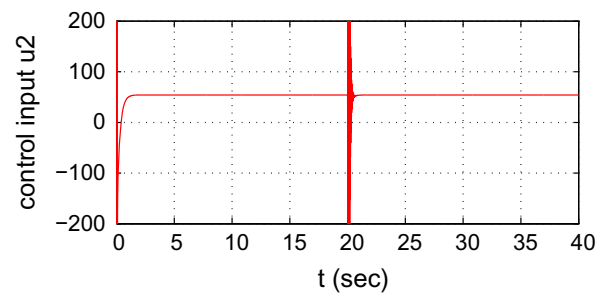

(b)

Fig. 4 Setpoint 3: a convergence of the state variables $x_{i}, i=1, \ldots, 4$ of the submarine to the desirable setpoints, $\mathbf{b}$ variations of the control inputs (bow and stern hydroplane reflections)
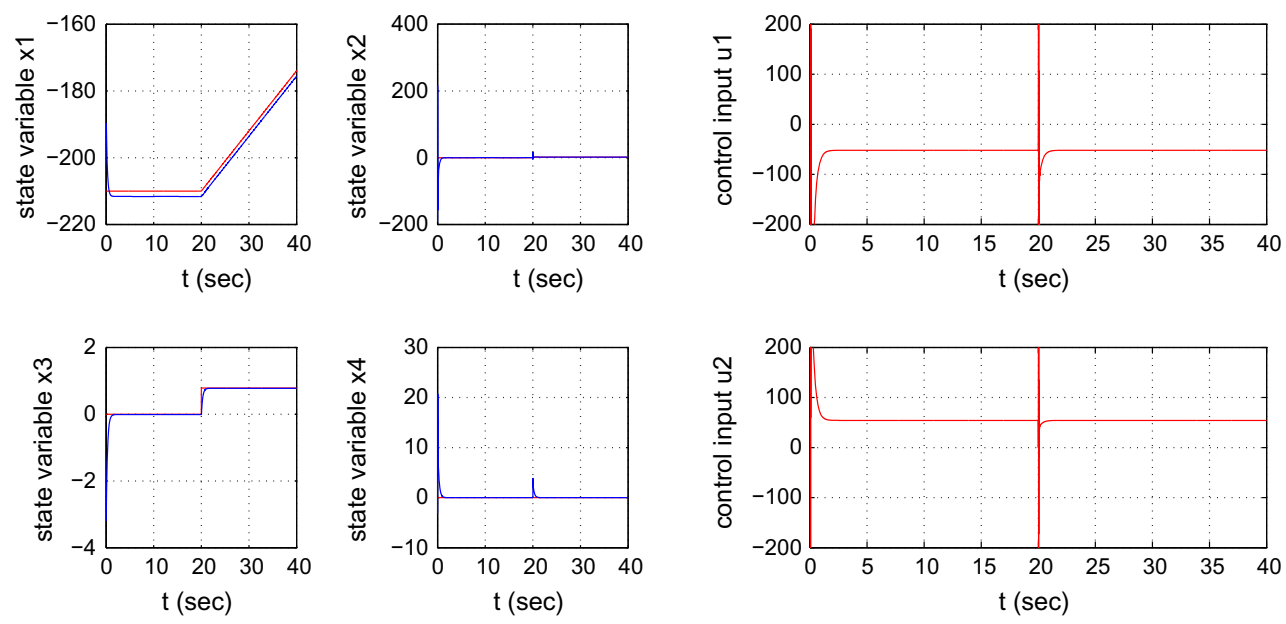

(a)

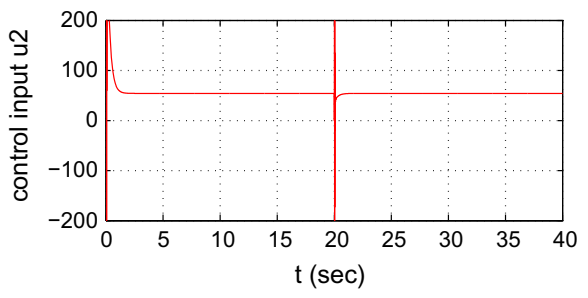

(b)

Fig. 5 Setpoint 4: a convergence of the state variables $x_{i}, i=1, \ldots, 4$ of the submarine to the desirable setpoints, $\mathbf{b}$ variations of the control inputs (bow and stern hydroplane reflections) 
tain system dynamics will be online identified by neurofuzzy approximators and that the state variables of the system will converge to the desirable setpoints. The robustness of the proposed adaptive fuzzy control method depends on the selection of parameters, such as the attenuation coefficient $\rho$ which is used in the solution of the associated Riccati equation.

Remark 7 The reference trajectories can be generated using the differential flatness properties of the system. This means that all state variables of the system are expressed as differential functions of the flat outputs. Next, reference trajectories are defined for the flat outputs and these are also used for computing the reference setpoints for the rest of the state variables of the submarines model.

\section{Conclusions}

The paper has proposed an adaptive fuzzy control method for autonomous submarines, that is based on differential flatness theory. By exploiting the differential flatness properties of the MIMO nonlinear model of the submarine the system was transformed into the linear canonical (Brunovsky) form. For the latter description of the system's dynamics the design of a feedback controller was possible. Moreover, to cope with unknown nonlinear terms appearing in the new control inputs of the transformed state-space description of the submarine, the use of neurofuzzy approximators has been proposed. These estimators were online trained to identify the unknown dynamics of the system and the associated learning procedure was determined by the requirement the derivative of the system's Lyapunov function to be a negative one. Moreover, through Lyapunov stability analysis it was proven that the closed loop satisfies the H-infinity tracking performance criterion, and this assures improved robustness against model uncertainties and external perturbations. Finally, the analytical results on the stability and robustness of the submarine's control loop were also confirmed through simulation experiments.

\section{References}

1. Do, K.D., Pan, J.: Global tracking control of underactuated ships with nonzero off-diagonal terms in their system matrices. Automatica 41, 87-95 (2005). Elsevier

2. Sira-Ramirez, H., Aguilar-Ibanez, C.: On the control of the hovercraft system. Dyn. Control 10, 151-163 (2000). Springer

3. Refsnes, J.B., Sorensen, A.J., Petersen, K.Y.: Model-based output feedback control of slender-body underactuated AUVs: theory and experiments. IEEE Trans. Control Syst. Technol. 16(5), 930-946 (2008)
4. Fischer, N., Hughes, D., Walters, P., Swartz, E.M., Dixon, W.E.: Nonlinear RISE-based control of an autonomous underwater vehicle. IEEE Trans. Robot. 30(4), 845-852 (2014)

5. He, B., Wang, B.R., Yan, T.H., Han, Y.Y.: A distributed parallel motion control for the multithruster autonomous underwater vehicle. Mech Based Des Struct Mach 41(2), 236-257 (2013). Taylor and Francis

6. Arslan, M.S., Fukushima, N., Hagiwara, J.: Nonlinear optimal control of an AUV and its actuator failure compensation. In: 10th International Conference on Control, Automation, Robotics and Vision, Hanoi, Vietnam (2008)

7. Fishcer, N., Bhasin, S., Dixon, W.E.: Nonlinear control of an autonomous underwater vehicle: a RISE-based approach. In: 2011 American Control Conference, San Francisco (2011)

8. Petersen, K.Y., Egeland, O.: Time-varying exponential stabilization of the position and attitude of an underactuated autonomous unerwater vehicle. IEEE Trans. Autom. Control 44(1), 112-115 (1999)

9. Rigatos, G.G.: Sensor fusion-based dynamic positioning of ships using extended Kalman and particle filtering. Robot. Camb. Univ. Press 31(3), 389-403 (2013)

10. Soltun, R.A., Ashrafiuon, H., Muske, K.R.: ODE-based obstacle avoidance and trajectory planning for unmanned surface vessels. Robotica 29, 691-703 (2010)

11. Lapierre, L., Saetanto, D.: Nonlinear path-following control of an AUV. Ocean Eng. 34, 1734-1744 (2007). Elsevier

12. Lapierre, L.: Robust diving control of an AUV. Ocean Eng. 36, 92-104 (2009). Elsevier

13. Moreira, L., Gueda Soares, C.: H2 and H1 design for diving and course control of an autonomous underwater vehicle in presence of waves. IEEE J. Ocean. Eng. 33(2), 69-88 (2008)

14. Liceaga-Castro, E., van der Molen, G.M.: Submarine H1 depth control under wave disturbances. IEEE Trans. Control Syst. Technol. 3(3), 338-346 (1995)

15. Sira-Ramirez, H.: Dynamic second-order sliding-mode control of the hovercraft vessel. IEEE Trans. Control Syst. Technol. 10(6), 860-865 (2002)

16. Lee, K.W., Singh, S.N.: Multi-input submarine control via L2 adaptive feedback despite uncertainties. J. Syst. Control Eng. 228(5), 330-347 (2014). Sage Publications

17. Pan, C.Z., Lai, X.Z., Yang, S.X., Wu, M.: An efficient neural network approach to tracking control of an autonomous surface vehicle with unknown dynamics. Expert Syst. Appl. 40, 1629-1635 (2013)

18. Zhang, L.J., Qi, X., Peng, Y.J.: Adaptive output feedback control based on DFRNN for AUV. Ocean Eng. 36, 716-722 (2009). Elsevier

19. Rigatos, G.G., Tzafestas, S.G.: Adaptive fuzzy control for the ship steering problem. J. Mechatron. 16(6), 479-489 (2006). Elsevier

20. Li, H.X., Tong, S.: A hybrid adaptive fuzzy control for a class of nonlinear MIMO systems. IEEE Trans. Fuzzy Syst. 11(1), 24-35 (2003)

21. Song, T., Bin, C., Wang, Y.: Fuzzy adaptive output feedback control for MIMO nonlinear systems. Fuzzy Sets Syst. 156, 285-299 (2005). Elsevier

22. Chen, C.S.: Dynamic structure adaptive neural fuzzy control for MIMO uncertain nonlinear systems. Inf. Sci. 179, 2676-2688 (2009). Elsevier

23. Tong, S., Li, Y.: Observer-based adaptive control for strict feedback nonlinear systems. Fuzzy Sets Syst. 160, 1749-1764 (2009)

24. Chen, C.H., Lin, C.M., Chen, T.Y.: Intelligent adaptive control for MIMO uncertain nonlinear systems. Expert Syst. Appl. 35, 865877 (2008)

25. Qi, R., Tao, G., Tan, C., Yao, X.: Adaptive control of discrete-time state-space TS fuzzy systems with general relative degree. Fuzzy Sets Syst. 217, 2240 (2013). Elsevier 
26. Yang, Y., Zhou, C., Jia, X.: Robust adaptive fuzzy control and its application to ship roll stabilization. Inf. Sci. 142(1-4), 177-194 (2002). Elsevier

27. Yousef, H.A., Hamdy, M., Shafiq, M.: Flatness-based adaptive fuzzy output tracking excitation control for power system generators. J. Frankl. Inst. 350(8), 2334-2353 (2013). Elsevier

28. Rigatos, G.: Nonlinear Control and Filtering Approaches Using Differential Flatness Theory: Applications to Electromechanical Systems. Springer, New York (2015)

29. Rigatos, G.: Modelling and Control for Intelligent Industrial Systems: Adaptive Algorithms in Robotics and Industrial Engineering. Springer, New York (2011)

30. Rigatos, G.: Advanced Models of Neural Networks: Nonlinear Dynamics and Stochasticity of Biological Neurons. Springer, Berlin (2013)

31. Rudolph, J.: Flatness Based Control of Distributed Parameter Systems, Examples and Computer Exercises from Various Technological Domains. Shaker, Aachen (2003)

32. Sira-Ramirez, H., Agrawal, S.: Differentially Flat Systems. Marcel Dekker, New York (2004)

33. Lévine, J.: On necessary and sufficient conditions for differential flatness. Appl. Algebra Eng. Commun. Comput. 22(1), 47-90 (2011). Springer

34. Fliess, M., Mounier, H.: Tracking control and $\pi$-freeness of infinite dimensional linear systems. In: Picci, G., Gilliam, D.S. (eds.) Dynamical Systems, Control, Coding and Computer Vision, vol. 258, pp. 41-68. Birkhauser, Berlin (1999)

35. Rouchon, P.: Flatness-based control of oscillators. J. Appl. Math. Mech. 85(6), 411-421 (2005). Wiley

36. Martin, Ph., Rouchon, P.: Syst"emes plats: planification et suivi des trajectoires, Journees XUPS, Ecole des Mines de Paris, Centre Automatique et Syst'emes, Mai (1999)
37. Bououden, S., Boutat, D., Zheng, G., Barbot, J.P., Kratz, F.: A triangular canonical form for a class of 0-flat nonlinear systems. Int. J. Control 84(2), 261-269 (2011). Taylor and Francis

38. Laroche, B., Martin, P., Petit, N.: Commande par platitude: Equations différentielles ordinaires et aux derivées partielles. Ecole Nationale Superieure des Techniques Avancées, Paris (2007)

39. Rigatos, G.: A differential flatness theory approach to observerbased adaptive fuzzy control of MIMO nonlinear dynamical systems. Nonlinear Dyn. 76(2), 1335-1354 (2014). Springer

40. Rigatos, G.G., Tzafestas, S.G.: Extended Kalman filtering for fuzzy modelling and multi-sensor fusion. Math. Comput. Model. Dyn. Syst. 13, 251-266 (2007). Taylor \& Francis

41. Basseville, M., Nikiforov, I.: Detection of Abrupt Changes: Theory and Applications. Prentice-Hall, Englewood Cliffs (1993)

42. Rigatos, G., Zhang, Q.: Fuzzy model validation using the local statistical approach. Fuzzy Sets Syst. 60(7), 882-904 (2009). Elsevier

43. Doyle, J.C., Glover, K., Khargonekar, P.P., Francis, B.A.: Statespace solutions to standard $\mathrm{H} 2$ and $\mathrm{H} 1$ control problems. IEEE Trans. Autom. Control 34, 831-847 (1989)

44. Kurylowicz, A., Jaworska, I., Tzafestas, S.G.: Robust stabilizing control : an overview. In: Tzafestas, S.G. (ed.) Applied Control: Current Trends and Modern Methodologies, pp. 289-324. Marcel Dekker, New York (1993)

45. Lublin, L., Athans, M.: An experimental comparison of and designs for interferometer testbed, Lectures Notes in Control and Information Sciences: Feedback Control. In: Francis, B., Tannenbaum, A. (eds.) Nonlinear Systems and Complexity, pp. 150-172. Springer, Berlin (1995)

46. Khalil, H.K.: Nonlinear Systems, 2nd edn. Prentice Hall, Upper Saddle River (1996)

47. Isidori, A.: Nonlinear Control Systems, 3rd edn. Springer, New York (1995) 